\title{
Trust the Literature, But Verify - Case Study of Starch Acetate as a Barrier Material Under Jungle Conditions
}

Peter W. Hart

A case study is presented in which several articles and patents suggested a specific outcome. When the actual experimental work was performed, the results were found to be several orders of magnitude away from predicted values. Close re-inspection of the literature suggested that most of the authors actually extrapolated the results to conditions that were not applicable to their specific studies, resulting in the reporting errors. It is important to use literature to assist in research, but it is equally important not to blindly follow it either.

Keywords: Starch Acetate; Literature; Patents; Barrier; Humidity conditions; WVTR

Contact information: Director: Research and Development, WestRock, Richmond, VA 23219 USA;

Email: peter.hart@westrock.com

\section{Age-Old Wisdom - Follow the Literature}

The librarian's dictum states that an hour in the library can save you six months in the lab. In general, I support this bit of wisdom. However, one must take care to fully understand the work being read in order to ensure the published work is truly relative to the current research problem being solved. In order to illustrate my point, I will use the case study of starch acetate as a barrier material under jungle conditions.

Several published articles and multiple patents discuss modified starches and specifically starch acetate as an excellent bio-polymeric barrier solution to replace several types of petroleum-derived options such as polyesters. The literature led us to believe that starches have excellent oxygen barrier properties and decent water barrier properties at low relative humidity $(\mathrm{RH})$. It was determined that starches are moisture-sensitive, causing the barrier properties to deteriorate at higher RH values. Several patents discuss the applicability of chemically modifying starches to make them barriers under high $\mathrm{RH}$ conditions. Specifically, starch acetates were listed as good coatings on paperboard for moisture barrier under multiple RH conditions.

Starch esters are chemically modified starches in which the hydroxyl group of the starch has been substituted by other moieties, such as acetates. This imparts hydrophobicity to starches and has been claimed as a good way of retaining water vapor barrier properties of starches even at high RH conditions. We studied the water vapor barrier properties of one class of starch esters, i.e. high amylose starch acetates (SA) that were assumed to have good water vapor barrier properties according to the literature.

Based upon these multiple and extremely positive literature reviews of chemically modified starch ester polymers as a barrier material, an extensive laboratory test was conducted. We carefully prepared, isolated, and purified starch acetate, starting with Hylon VII High Amylose Starch (HAS) from Ingredion Inc. The purified starch acetate was plasticized with $20 \mathrm{wt}$ percent glyceryl triacetate and cast into a film. The chemical composition (free hydroxyl group content) of the free-standing films as well as HAS 
powder was qualitatively analyzed using a Thermo Scientific Nicolet 6700 Fourier Transform Infrared (FTIR) spectrometer with SensIR Durascope diamond attenuated total reflectance setup to ensure we had succeeded in preparing starch acetate films. The prepared films were tested at jungle conditions (high $\mathrm{RH}$ ) for water vapor transmission rate (WVTR) using the jar method (TAPPI T464om-2012) to obtain WVTR over 48 hours. We prepared SA films with two distinct levels of chemical substitution, one with almost no free $\mathrm{OH}$ groups and one with a lesser degree of substitution. Based upon literature results, we expected a low WVTR of the starch tri-acetate (STAc) over the starch bi-acetate (SBAc) due to the lower number of $\mathrm{OH}$ groups in the STAc.

\section{The Reveal - Or What the Heck Happened?}

It was expected that SA films would display WVTR values similar to those of commercial polyesters. However, it was seen that the WVTR of both kinds of SA films was much higher than values reported in the literature. The STAcs, having almost no $\mathrm{OH}$ groups, had lower WVTR than SBAc (which was expected from lower number of $\mathrm{OH}$ groups), yet had WVTR which was orders of magnitude higher than literature reported values for starch acetates, polyesters, and polyamides. Despite patent literature pointing to their application as moisture barrier materials, this investigation showed that these materials do not perform well as barrier materials. As such, while biodegradable and economically competitive, they are still not comparable to LDPE as water vapor barriers.

\section{Take Literature with a Grain of Salt}

Upon closer inspection of the literature sources, most of the references gave actual WVTR values at typical room conditions and simply stated the applicability of the barrier application at high HR conditions. Only one literature source independently provided WVTR rates for these films under jungle conditions. Many of the patents stated applicability but had not actually tested materials under high RH conditions. As such, even though they clearly stated the applicability of SA films as moisture barriers under multiple conditions, including jungle conditions, they had never actually tested them under those conditions. Rather, the results were extrapolated beyond the viability of the existing data to obtain and report erroneous results.

In general, the work reported in the literature was of good quality where reported data were actually available. Several literature reviews reported the WVTR values for standard conditions then expounded upon the benefits of chemical modification and led readers to believe these materials worked at high $\mathrm{RH}$ conditions as well. Several of the patents simply reported high $\mathrm{RH}$ conditions for barrier properties to carve out the space without ever performing a single experiment under those conditions.

This case study shows the value and the limitations of using literature studies to help direct research. It is important to verify the conditions that were actually tested and reported upon in literature studies. It is also important to determine where the authors have extrapolated well beyond the existing experimental structure and postulated potentially questionable results. 